\title{
Perfusión aislada de extremidad: una opción terapéutica para metástasis en tránsito de melanoma nodular y lentiginoso acral
} Isolated limb perfusion: $\boldsymbol{A}$ therapeutic option for nodular and acral lentiginous melanoma

\author{
Carlos Duarte', Juliana Restrepo*, Alden Pool Gómez ${ }^{3}$ y Alexander Carreño4
}

${ }^{1}$ Servicio de Seno y Tejidos Blandos, Instituto Nacional de Cancerología, ESE, Asociación Colombiana de Mastología, Universidad del Rosario, Universidad Militar Nueva Granada, Bogotá; ${ }^{2}$ Servicio de Cirugía Oncológica. Instituto Nacional de Cancerología, ESE, Universidad Militar Nueva Granada, Bogotá; ${ }^{3}$ Servicio de Cirugía Oncológica. Hospital Universitario del Valle, Universidad del Valle, Cali; ${ }^{4}$ Grupo de Investigación Clínica. Instituto Nacional de Cancerología, ESE, Bogotá. Colombia

\section{Resumen}

Objetivo: describir la experiencia inicial en Colombia en el manejo del melanoma de extremidad con perfusión aislada y comparar la respuesta de los subtipos histológicos más frecuentes en Colombia con la literatura. Materiales y métodos: estudio descriptivo, retrospectivo de una serie de pacientes con diagnóstico de melanoma con metástasis en tránsito tratados con perfusión aislada de extremidad, seleccionados entre 2007 y 2016. Las variables cualitativas se analizaron con frecuencias absolutas y relativas. Las cuantitativas con medidas de tendencia central y dispersión. Resultados: 11 pacientes fueron tratados con perfusión aislada de extremidad. Hubo respuesta parcial en siete pacientes (63,6\%), enfermedad estable en dos pacientes (18,2\%) y progresión en dos pacientes (18,2\%). Después del tratamiento, cinco pacientes (45,5\%) presentaron complicaciones menores. Conclusiones: la perfusión aislada sigue siendo importante para el tratamiento del melanoma localmente avanzado con el fin de mejorar la calidad de vida de los pacientes. La respuesta puede cambiar según el tipo clínico de melanoma, siendo menor, según este estudio, en pacientes con melanoma lentiginoso acral y nodular, aunque por el bajo poder, esto no se puede concluir.

Palabras clave: Melanoma. Quimioterapia. Cáncer. Perfusión regional. Salvamento de extremidad.

\begin{abstract}
Objective: to describe the initial experience in Colombia of locally advanced melanoma treated by isolated limb perfusion and compare the response rate of our melanoma subtypes with the literature. Methods: descriptive, retrospective study of a series of patients selected between 2007 and 2016. Qualitative variables were analyzed with proportions and frequencies, and quantitative variable with central tendency measures and measures of dispersion. Results: 11 patients were treated by isolated limb perfusion. After the treatment, seven patients (63.6\%) had partial response, two patients (18.2\%) had stable disease and two patients (18.2\%) had disease progression. Five patients (45.5\%) had mild complications. Conclusions: Isolated limb perfusion is still an important treatment strategy for locally advanced melanoma, with the goal of improving the patient quality of life. The response can change according to the type of melanoma, and could be smaller in patients with acral lentiginous and nodular melanoma, although we cannot make this conclusion with this type of study because it has low power and lack of a comparison group.
\end{abstract}

Key words: Melanoma. Chemotherapy. Cancer. Regional perfusion. Limb salvage.

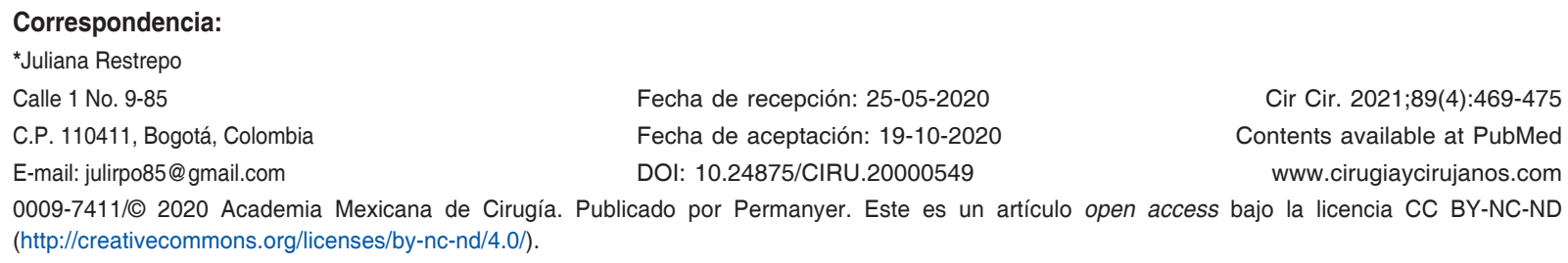




\section{Introducción}

La perfusión aislada de extremidad (PAE) es una alternativa de tratamiento en pacientes con melanoma localmente avanzado de extremidades, en el cual se obtienen altas concentraciones de los medicamentos quimioterapéuticos en la extremidad comprometida. Su principal objetivo es disminuir la carga tumoral y preservar la extremidad. Se debe evitar la toxicidad sistémica mediante una técnica quirúrgica rigurosa ${ }^{1-3}$.

A pesar de que en el mundo existen centros con más de 50 años de experiencia en este tratamiento ${ }^{4,5}$, existen pocos estudios reportando la experiencia en América Latina ${ }^{6}$. En algunos países como Colombia la incidencia de ciertos subtipos de melanoma como el lentiginoso acral es mayor y por ende la respuesta a la perfusión aislada de extremidad puede tener un comportamiento diferente, cuando se compara con los resultados de países con predominio de otros subtipos clínicos.

El presente estudio describe los resultados de la PAE en pacientes con melanoma con metástasis en tránsito (MET) tratados en el Instituto Nacional de Cancerología (INC) en Bogotá, Colombia. El objetivo del estudio es describir nuestra experiencia inicial y comparar los resultados con otros países en América Latina y el mundo.

\section{Metodología}

Estudio descriptivo, retrospectivo en el que se realizó una búsqueda activa del sistema electrónico de historias clínicas (SAP) del INC. Se seleccionaron pacientes con diagnóstico de melanoma de extremidad con MET tratados con PAE entre enero de 2007 y enero de 2016. Los casos identificados debían cumplir con los siguientes criterios de inclusión: confirmación histopatológica de melanoma, melanoma del cualquier subtipo clínico con MET, que hubieran recibido tratamiento con PAE con intención curativa 0 paliativa.

De cada historia clínica se obtuvieron las variables sociodemográficas edad y sexo. Entre las variables clínicas se incluyeron la localización, el subtipo clínico del melanoma, el breslow, el estado ganglionar y la etapa clínica al momento del diagnóstico. Entre las variables de resultado del procedimiento se incluyeron el tiempo operatorio, la estancia hospitalaria y en la Unidad de Cuidado Intensivo (UCI), las complicaciones y la respuesta al tratamiento según los criterios de la Organización Mundial de la Salud (Respuesta Completa, Parcial, Enfermedad estable y Progresión de la enfermedad). Igualmente se obtuvieron datos sobre la toxicidad al tratamiento, la cual fue clasificada de acuerdo a la escala de Wieberdink?

Los datos se recolectaron en Excel (Microsoft Corp) y fueron analizados utilizando SPSS 22 según la naturaleza de las variables; para la cualitativas se realizo un análisis en términos de proporciones y frecuencias, en cuanto a las variables cuantitativas se determino si su distribución era normal, y fueron analizadas según el caso en términos de medidas de tendencia central y dispersión.

Se obtuvo aprobación del comité de ética del Instituto Nacional de Cancerología.

\section{Descripción de la técnica: perfusión aislada de la extremidad}

Todos los pacientes recibieron tratamiento con PAE bajo anestesia general. Se utilizó monitoria invasiva, catéter venoso central y línea arterial. Inicialmente se disecaron las arterias y las venas principales de la extremidad afectada proximales al tumor. Se disecan en un trayecto de $5 \mathrm{~cm}$ ligando todas sus colaterales y el aislamiento de la extremidad se efectúa con un torniquete neumático, a una presión de $250 \mathrm{~mm} \mathrm{Hg}$. Posteriormente se cateterizaron la arteria y la vena principales. En el miembro superior las localizaciones anatómica susceptibles de intervención son los vasos axilares o humerales y en el miembro inferior son los vasos ilíacos, femorales comunes, femorales superficiales y poplíteos. La arteriotomía y la venotomía se hacieron de forma transversa, para disminuir el riesgo de estenosis. La arteria y la vena se canalizaron mediante cánulas, a un circuito de circulación extracorpórea que incluye un oxigenador y un calentador que mantiene el sistema a $41^{\circ} \mathrm{C}$.

Se inyectaron en el circuito pirofosfatos, los cuales tienen como objetivo sensibilizar los glóbulos rojos al tecnecio 99 que se infundirá posteriormente. Con una gamma sonda en la región precordial se descartó la presencia de fugas hacia la circulación general y se administró Melphalan en una dosis de $10 \mathrm{mg} / \mathrm{lt}$ de extremidad perfundida para lesiones de miembro inferior y de $13 \mathrm{mg} / \mathrm{lt}$ para lesiones de miembro superior. La concentración de Melphalan es 10 a 20 veces más alta en el circuito aislado en comparación con una dosis sistémica usual. La perfusión aislada se realizó durante 60 minuto, se retiraron los catéteres y se repararom los vasos. 


\section{Resultados}

Once pacientes fueron incluidos, la mediana de edad fue de 59 años (rango 49 a 67 años), ocho (72.7\%) fueron mujeres y en nueve casos $(81.8 \%)$ el compromiso fue en miembros inferiores. En la clasificación de los subtipos clínicos siete pacientes $(63.6 \%)$ tenían melanoma lentiginoso acral y cuatro (36.4\%) tenían melanoma nodular. La mediana del breslow (Rango) fue de $3.6 \mathrm{~mm}(1.1-20)$ y 8 pacientes $(72.7 \%)$ tenían ganglios positivos y metástasis en trásnsito al momento del diagnóstico (Tabla 1).

La mediana de tiempo operatorio (Rango) fue de 267.5 (105 - 375) minutos, la mediana de días de estancia hospitalaria (rango) fue de 6.5 (1-17) días y de estancia en $\mathrm{UCl}$ (rango) fue de 2 (2.-5) días (Tabla 2).

Con la perfusión aislada de la extremidad se obtuvo respuesta parcial en siete pacientes $(63.6 \%)$, enfermedad estable en dos pacientes (18.2\%) y progresión de la enfermedad en dos pacientes (18.2\%) (Tabla 2).

La mediana de supervivencia fue de 17 meses, la probabilidad de supervivencia a 1 año fue del $70.7 \%$ y a 33 meses del $36.4 \%$ (Fig. 1). Cuatro pacientes (36.4\%) presentaron recaída (dos hombres y dos mujeres) y nueve pacientes $(81.8 \%)$ fallecieron en un seguimiento promedio de 18 meses (rango 3 a 30 meses) (Tabla 2).

Las complicaciones posteriores a la PAE se clasificaron según Wieberdink ${ }^{7}$ (Tabla 2). Se observó edema leve en ocho pacientes (72.7\%), edema moderado en uno (9.1\%). Como complicaciones posquirúrgicas tardías se observaron dos casos (18.2\%) de infección del sitio operatorio y un caso de trombosis venosa profunda $(9.1 \%)$. Desde el punto de vista técnico hubo fuga del agente antineoplásico en un procedimiento (9.1\%). (Tabla 2). No hubo ningún caso de toxicidad sistémica severa ni mortalidad por el procedimiento.

\section{Discusión}

A nivel global la incidencia de melanoma sigue aumentando ${ }^{8}$. En América Latina, la información epidemiológica del melanoma es limitada y sólo se describen experiencias institucionales ${ }^{9-12}$. En una revisión epidemiológica publicada en Brasil en el 2011 se encontró que la incidencia es menor que la de regiones más desarrolladas como Europa y Norte América ${ }^{13}$.

\begin{tabular}{lllc} 
Tabla 1. Características demográficas y clínicas de los pacientes \\
\hline Variables & Descripción & N & $\begin{array}{c}\text { Número (\%) o } \\
\text { mediana (rango) }\end{array}$ \\
\hline Sexo & Masculino & 3 & 27.3 \\
& Femenino & 8 & 72.7 \\
Edad promedio (rango) & 59 & 11 & $49-67$ \\
Localización & Miembro & 2 & 18.2 \\
& superior & & \\
& Miembro & 9 & 81.8 \\
& inferior & & \\
Clasificación & Lentiginoso & 7 & 64 \\
& acral & & \\
& Nodular & 4 & 36 \\
Breslow al diagnóstico & Milímetros & 11 & $3.6(1.1-20)$ \\
Estado ganglionar al & Negativo & 3 & 27.3 \\
diagnóstico & Positivo & 8 & 72.7 \\
Etapa clínica al diagnóstico & I & 1 & 9.1 \\
& II & 2 & 18.2 \\
& III & 8 & 54.5 \\
\hline
\end{tabular}

*Según la estadificación AJCC 7ma ed.

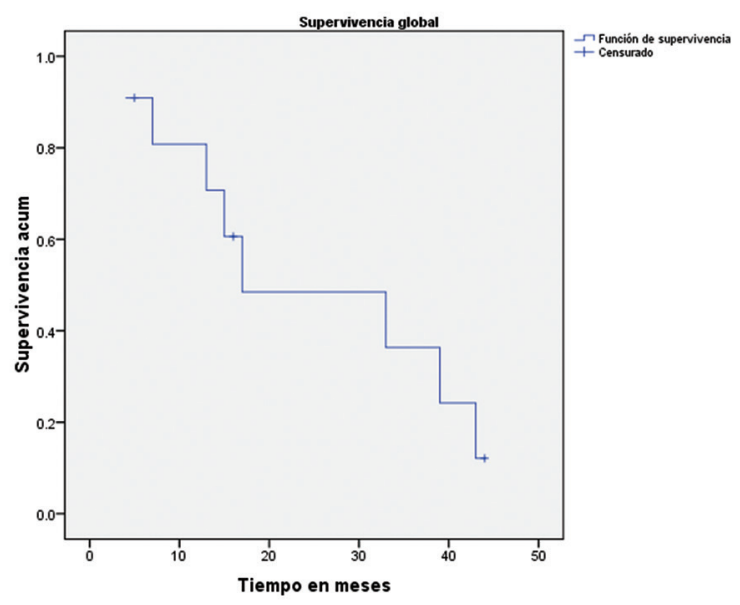

Figura 1. Tabla de Kaplan Meier que describe la supervivencia de la cohorte de pacientes con metástasis en tránsito de melanoma tratados con PAE.

PAE: Perfusión Aislada de Extremidad.

En Colombia algunos estudios han mostrado un relativo incremento en la incidencia y el patrón clínico predominante es el lentiginoso acral ${ }^{9,14}$. En un estudio publicado en el 2018 por una institución privada en Colombia $^{12}$, el melanoma de extensión superficial representó el $42 \%$, lentigo maligno $31 \%$ y el lentiginoso acral el $21 \%$. A diferencia de un estudio previo publicado en el Instituto Nacional de Cancerología ${ }^{9}$, una institución pública, donde la población es similar a la 
Cirugía y Cirujanos. 2021;89(4)

Tabla 2. Seguridad del procedimiento y respuesta al tratamiento

\begin{tabular}{|c|c|c|c|}
\hline Variable & Descripción & $\mathbf{N}$ & $\begin{array}{c}\text { Número (\%)/ } \\
\text { mediana (rango) }\end{array}$ \\
\hline Tiempo operatorio & Minutos & 11 & $267.5(105-375)$ \\
\hline Estancia hospitalaria & Días & 11 & $6.5(1-17)$ \\
\hline Unidad de Cuidados Intensivos & Días & 11 & $2(0-5)$ \\
\hline Fallo de la perfusión & $\begin{array}{l}\text { No } \\
\mathrm{Si}\end{array}$ & $\begin{array}{l}9 \\
2\end{array}$ & $\begin{array}{l}81.8 \\
18.2\end{array}$ \\
\hline Complicaciones durante la PAE & Fuga del agente & 1 & 9.1 \\
\hline Toxicidad por perfusión según la escala de Wieberdink & $\begin{array}{l}\text { I } \\
\text { II } \\
\text { III } \\
\text { IV } \\
\text { V }\end{array}$ & $\begin{array}{l}2 \\
8 \\
1 \\
0 \\
0\end{array}$ & $\begin{array}{c}18.2 \\
72.7 \\
9.1 \\
0.0 \\
0.0\end{array}$ \\
\hline Complicaciones quirúrgicas posteriores & $\begin{array}{l}\text { ISO* superficial } \\
\text { ISO* profunda } \\
\text { Déficit neurológico }\end{array}$ & $\begin{array}{l}2 \\
1 \\
1\end{array}$ & $\begin{array}{l}18.2 \\
9.1 \\
9.1\end{array}$ \\
\hline Complicaciones sistémicas & $\begin{array}{l}\text { Trastorno hidroelectrolítico } \\
\text { Trastornos pulmonares }\end{array}$ & $\begin{array}{l}1 \\
1\end{array}$ & $\begin{array}{l}9.1 \\
9.1\end{array}$ \\
\hline Respuesta a la perfusión & $\begin{array}{l}\text { Parcial } \\
\text { Completa } \\
\text { Estable } \\
\text { Progresión }\end{array}$ & $\begin{array}{l}7 \\
0 \\
2 \\
2\end{array}$ & $\begin{array}{c}63.6 \\
0.0 \\
18.2 \\
18.2\end{array}$ \\
\hline Respuesta al tratamiento & $\begin{array}{l}\text { Recaída } \\
\text { Salvamento de la extremidad }\end{array}$ & $\begin{array}{c}4 \\
11\end{array}$ & $\begin{array}{c}36.4 \\
100\end{array}$ \\
\hline Supervivencia a 18 meses & $\begin{array}{l}\text { Vivos } \\
\text { Muertos }\end{array}$ & $\begin{array}{l}2 \\
9\end{array}$ & $\begin{array}{l}18.2 \\
81.8\end{array}$ \\
\hline
\end{tabular}

ISO: infección del sitio operatorio.

población general colombiana y donde el patrón predominante fue el lentiginoso acral, que representaba el $45 \%$ de la serie. Esto se puede explicar porque la serie publicada en el 2018 tiene predominio de fototipos de Fitzpatrick I a III, fototipos que predominan en las personas con altos ingresos que utilizan los sistemas privados de salud.

En los estudios anglosajones predomina el patrón de crecimiento de extensión superficial ${ }^{8}$ y en nuestra institución el melanoma lentiginoso $\mathrm{acral}^{9}$, por esta razón consideramos que es importante conocer los resultados de la PAE en la población del INC donde predomina el patrón clínico lentiginoso acral.

En América Latina el primer reporte de PAE para el tratamiento de melanoma fue publicado en Brasil en el 2008, incluyeron 41 pacientes y obtuvieron una respuesta completa en $43 \%$ y una respuesta parcial en $36 \%$ pero no hay descripción de la distribución de los subtipos clínicos ${ }^{6}$. En el 2007 se implementó por primera vez esta técnica en Colombia para el manejo de tumores en extremidades y en una publicación en el 2011 se reportaron los resultados en pacientes con diagnóstico de melanoma, sarcoma y carcinoma de anexos cutáneos ${ }^{15}$.

En pacientes con diagnóstico de melanoma las metástasis en tránsito se presentan en estados avanzados de la enfermedad y se consideran una condición clínica de mal pronóstico. Desde el punto de vista terapéutico la resección quirúrgica de las metástasis en tránsito es un tratamiento capaz de mejorar los síntomas y la alteración de la función de la extremidad afectada', pero algunos pacientes desarrollan múltiples lesiones o lesiones de gran tamaño que no son susceptibles de resección quirúrgica'. En pacientes con melanoma de extremidad localmente avanzado, irresecable, la PAE tiene múltiples estudios acerca de su efectividad y toxicidad ${ }^{1}$, este tratamiento puede evitar una amputación y mejorar la calidad de vida de los pacientes con esta patología. La elección de la terapia depende del número de lesiones, su 
localización anatómica, el tamaño y la presencia o ausencia de enfermedad extra regional'16,17. La amputación casi nunca está indicada y no mejora la supervivencia en estos pacientes ${ }^{18}$.

En este estudio la respuesta es menor a la reportada en la literatura, con una respuesta parcial en dos tercios de los pacientes y enfermedad estable en el $18.2 \%$. Diferentes estudios han publicado tasas de respuesta entre 80 y 90\%1,19 y los reportes de respuesta completa están alrededor del 50\%3,19,20. Estas publicaciones son heterogéneas en cuanto a la carga tumoral y la distribución de los subtipos clínicos varía según la localización demográfica, esto podría explicar las diferentes tasas de respuesta. Aún así, la respuesta sigue siendo mayor a la respuesta del melanoma metastásico a la quimioterapia citotóxica sistémica la cual oscila entre 15 y $46 \%$, sin impactar en la supervivencia global ${ }^{20,21}$. Un estudio publicado por Belgrano y col en el 2019, reportó el uso repetido de infusión de la extremidad, con respuesta completa de $60 \%$ en la primera sesión, $41 \%$ y $59 \%$ en las subsecuentes $^{22}$. En este estudio no se realizaron perfusiones múltiples en un mismo paciente.

En el presente estudio nueve pacientes fallecieron a los 18 meses y aunque a la fecha ningún tratamiento locoregional para las metástasis en tránsito de melanoma ha tenido impacto en la supervivencia global', en esta publicación la supervivencia es menor a la reportada en la literatura, la cual es $29 \%$ a 5 años ${ }^{3}$, posiblemente porque predominan los melanomas de tipo lentiginoso acral y nodular cuya biología tumoral es más agresiva ${ }^{14,23}$.

Las recurrencias locales están descritas entre $40 \%$ y $50 \%$ a 6 meses después del tratamiento ${ }^{1,3,24}$, resultados similares a los encontrados en paciente con metástasis en tránsito resecadas quirúrgicamente. En este estudio las recurrencias locales se presentaron en el $36.3 \%$ de los pacientes durante una mediana de seguimiento de 18 meses, no es posible hacer la comparación a 6 meses porque los datos no están disponibles. En una revisión sistemática de estudios aleatorizados no se encontró beneficio con el uso de PAE profiláctica ${ }^{20}$.

La toxicidad encontrada en nuestra serie fue similar a la descrita en la literatura ${ }^{1,15,25}$. Un estudio publicado en Brasil en el $2012^{2}$ reportó toxicidad de la PAE en 46 pacientes con diagnóstico de melanoma; la perfusión se realizó con Melphalan al igual que en nuestra serie y se encontró que la toxicidad grado I fue de $2 \%$, grado II $78 \%$, grado III $7 \%$, grado IV $5 \%$ y grado V $9 \%$. En nuestro estudio la toxicidad grado II fue también la más frecuente pero no hubo toxicidades grado IV ni V. Los factores asociados a mayor toxicidad según las diferentes publicaciones son la temperatura mayor o igual a $40^{\circ} \mathrm{C}, \mathrm{pH}$ acido, hipoxia y valores de hematocrito menores a $20 \% 3,26$.

La realización de la PAE evitó la amputación de la extremidad en la totalidad de los paciente, en la literatura las tasas de salvamento de extremidad son similares alrededor del $97 \%{ }^{3}$ y en una revisión sistemática de estudios aleatorizados la tasa de amputación fue de 0,005\% ${ }^{20}$.

Los estudios como el nuestro utilizan Melphalan como medicamento para la PAE, pero también se ha utilizado el factor de necrosis tumoral alfa (FNT $\alpha$ ) el cual aumenta la permeabilidad vascular intratumoral mejorando la biodisponibilidad del medicamento citostático. Recientes investigaciones evaluaron el uso de la combinación del FNT $\alpha$ y el Melphalan obteniendo respuestas completas de la enfermedad en un $80 \%{ }^{3,24,27-30}$, pero el estudio ACOSOG Z002025 comparó la efectividad del FNT $\alpha$ más Melphalan con Melphalan sólo, en pacientes con melanoma de extremidades y metástasis en tránsito. Después de un análisis interino en el 2004, decidieron suspender el estudio porque no hubo diferencias en la respuesta entre los dos grupos, pero si mayor toxicidad en el grupo de pacientes que recibieron FNT $\alpha$. Sin embargo muchos centros continúan recomendando el uso del TNF alfa asociado a Melphalan en pacientes con grandes volúmenes tumorales, o en pacientes que son candidatos a una segunda perfusión ${ }^{3}$. Pero este medicamentos no está disponible en Colombia.

Para futuros estudios, definir el papel de la PAE en la era actual de la inmunoterapia va a ser de gran importancia. Los tratamientos loco-regionales pueden causar la muerte de células inmunogénicas del tumor y otros eventos inmunogénicos favorables. Algunos estudios en modelos animales, además de datos anecdóticos de casos humanos, sugieren que las terapias regionales puedan tener un efecto abscopal distante a la región tratada ${ }^{31}$. El melanoma expuesto a Melphalan estimula la expansión de monocitos $C D$ $16+$ y activa células $T$ citotóxicas que contribuyen a la eficacia antitumoral de la PAE. Además, la muerte de células tumorales durante la PAE resulta en la liberación de grandes cantidades de antígenos que aumentan la respuesta de las células $\mathrm{T}^{32}$. Los paciente en el presente estudio se trataron cuando no estaban disponibles aún la inmunoterapia y las terapias dirigidas. La sorprendente mejoría en los resultados de los pacientes con melanoma con estas terapias 
sistémicas durante los últimos años hace que sea esencial estudiar el efecto abscopal de las terapias locales en combinación con las terapias sistémicas ${ }^{33}$. Algunos estudios concluyen que el uso de tratamientos regionales como la PAE y la infusión aislada en pacientes con melanoma con MET siguen siendo un estrategia útil porque tienen una respuesta buena y duradera ${ }^{34}$.

No fue posible evaluar la calidad de vida de los pacientes porque los datos no se encontraron en las historias clínicas, esta es una de las limitantes más frecuentes de los estudios retrospectivos. Un estudio publicado en el 2019 reportó que los pacientes con metástasis en tránsito van en múltiples ocasiones a resecciones quirúrgicas con alta morbilidad y recaídas tempranas, en cambio, con el tratamiento con infusión o perfusión? de la extremidad, los paciente y médicos? reportaron un disminución sostenida de la carga tumoral y por ende una mejor calidadvida ${ }^{35}$.

Este estudio tiene como limitaciones que es una cohorte pequeña de pacientes con datos recolectados retrospectivamente por lo que no fue posible encontrar en las historias clínicas el número de metástasis en tránsito. Adicionalmente, no fue posible encontrar los datos en algunas variables como el tiempo operatorio de dos pacientes y el breslow de un paciente. No hay grupo comparativo lo que hace que las comparaciones se deban hacer con otros artículos de la literatura y se realizó en un época en la que la inmunoterapia no estaba disponible, lo que hace que no se pueda evaluar la interacción de estos dos tratamientos.

\section{Conclusión}

La PAE sigue siendo una herramienta importante para el tratamiento de melanoma de extremidad con metástasis en tránsito no resecables con el fin de mejorar la funcionalidad y la calidad de vida de los pacientes. No se puede esperar que la PAE terapéutica mejore la supervivencia o disminuya la progresión sistémica de la enfermedad. La respuesta puede cambiar según el tipo clínico de melanoma, siendo menor en pacientes con melanoma lentiginoso acral y melanoma nodular comparando este estudio con la literatura mundial, aunque por el bajo poder de este estudio y la falta de un grupo de comparación no se puede definir la asociación entre la respuesta a la PAE y el tipo clínico de melanoma. Finalmente, los pacientes con melanoma localmente avanzado irresecable son candidatos a inmunoterapia y es poco probable que una terapia regional se vaya a utilizar sola en pacientes con enfermedad extensa; esto abre las puertas para el uso combinado de PAE que proporciona una respuesta rápida en pacientes con alta carga tumoral en extremidades asociada a inmunoterapia que mejora la supervivencia. Se requieren más estudios para definir si estas dos terapias combinadas pueden mejorar los desenlaces de estos pacientes.

\section{Financiamiento}

La presente investigación no ha recibido ayudas específicas provenientes de agencias del sector público, sector comercial o entidades sin ánimo de lucro.

\section{Conflicto de intereses}

Los autores no declaran ningún conflicto de intereses.

\section{Responsabilidades éticas}

Protección de personas y animales. Los autores declaran que para esta investigación no se han realizado experimentos en seres humanos ni en animales.

Confidencialidad de los datos. Los autores declaran que han seguido los protocolos de su centro de trabajo sobre la publicación de datos de pacientes.

Derecho a la privacidad y consentimiento informado. Los autores han obtenido el consentimiento informado de los pacientes y/o sujetos referidos en el artículo. Este documento obra en poder del autor de correspondencia.

\section{Bibliografía}

1. Moreno-Ramirez D, de la Cruz-Merino L, Ferrandiz L, Villegas-Portero R, Nieto-García A. Isolated Limb Perfusion for Malignant Melanoma: Systematic Review on Effectiveness and Safety. Oncologist. 2010;15:416427. doi:10.1634/theoncologist.2009-0325

2. Neto D, Oliveira IF, Bertolli IIE, et al. Isolated limb perfusion with hyperthermia and chemotherapy: predictive factors for regional toxicity. 2012;67(3):237-241. doi:10.6061/clinics/2012(03)06

3. Kroon BBR, Noorda EM, Vrouenraets BC, Slooten GW Van, Nieweg OE. Isolated Limb Perfusion for Melanoma. 2008;17:785-794. doi:10.1016/j. soc.2008.04.005

4. Creech O, Krementz E, Ryan RF, Winblad JN. Chemotherapy of Cancer : Regional Perfusion Utilizing an Extracorporeal Circuit. Ann Surg. 1958;148(4):616-632.

5. Colomb FM, Postel AH, Hall AB, Gumport SL, Cox KR, C WJ. Chemotherapy of Human Cancer by Regional Perfusion. Cancer. 1962;15(4):828845.

6. Pavan Pasin V, Fernandes De Oliveira A, Oliveira Santos ID de A, Masako Ferreira L. Hyperthermic Isolated Limb Perfusion in the Treatment of In-Transit Melanoma Metastases Victor. Rev Col Bras Cir. 2008;35(3):159-161. 
7. Wieberdink J, Benckhuysen C, Braat R, Van Slooten E, Olthuis A. Dosimetry in Isolation Perfusion of the Limbs by Assessment of Perfused Tissue Volume and Grading of Toxic Tissue Reactions. Eur J Cancer Clin Oncol. 1982;18(10):905-910.

8. Erdei E, Torres SM. A new understanding in the epidemiology of melanoma. Expert Rev Anticancer Ther. 2010;10(11):1811-1823.

9. Pozzobon F, Acosta A, Carreño A, Fierro E. Características del melanoma cutáneo primario en el Instituto Nacional de Cancerología 2006-2010. Rev Colomb Cancerol. 2013;17(3):111-118.

10. Ferrari Junior NM, Muller H, Ribeiro M, Maia M, Sanchez Junior JA. Cutaneous melanoma: descriptive epidemiological study. Sao Paulo Med J. 2008;126(1):41-47.

11. Gutiérrez C, Alarcón E, Valle R, Carderón G. Epidemiología del melanoma maligno en el Instituto Nacional de Enfermedades Neoplásicas, Perú, 2000-2004. Folia Dermatol Peru. 2007;18(1):23-27.

12. Pozzobon FC, Acosta AE. Epidemiological profile of primary cutaneous melanoma over a 15-year period at a private skin cancer center in CoIombia. Rev Salud Pública. 2018;20(2):226-231.

13. Sortino-Rachou AM, Curado MP, Cancela M de C. Cutaneous melanoma in Latin America: a population-based descriptive study. Cad Saude Publica. 2011;27(3):565-572. doi:10.1590/S0102-311X2011000300016

14. Colmenares Roldán LM, Velásquez Lopera M, Vargas Suaza GA. Melanoma lentiginoso acral : una variante de melanoma maligno de especial interés en Colombia. latreia. 2008;21(4):386-397.

15. Duarte C, García M, Lehmann C, Ricardo S, Manrique J. Perfusión aislada de extremidades. Experiencia inicial del Instituto Nacional de Cancerología, 2007-2008. Rev Colomb Cancerol. 2011;15(2):67-74.

16. Fields RC, Fleming MD, Gastman B, et al. NCCN Melanoma Guidelines 2017. 2017;1.

17. Fink L, Amelio J, Harries M, Lebbe C, Livingstone E, Flinois A. Making decisions on resectability and injectability status of lesions in the management of advanced melanoma in Germany, France and the UK. Eur $J$ Dermatol. 2016;26(5):477-486. doi:10.1684/ejd.2016.2821

18. Grünhagen DJ, de Wilt JHW, van Geel AN, Eggermont AMM. Isolated limb perfusion for melanoma patients - a review of its indications and the role of tumour necrosis factor- $\alpha$. Eur J Surg Oncol. 2006;32(4):371-380. doi:10.1016/j.ejso.2006.01.015

19. Weitman ES, Zager JS. Regional therapies for locoregionally advanced and unresectable melanoma. Clin Exp Metastasis. 2018;0(0):0. doi:10.1007/s10585-018-9890-1

20. Lens MB, Dawes M. Review Isolated limb perfusion with melphalan in the treatment of malignant melanoma of the extremities : a systematic review of randomised controlled trials. Lancet Oncol. 2003;44:359-364.

21. Li Y, Mcclay EF. Systemic Chemotherapy for the Treatment of Metastatic Melanoma. Semin Oncol. 2002;29(5):413-426. doi:10.1053/sonc.2002.35237

22. Belgrano V, Pettersson J, Nilsson JA, Mattsson J, Katsarelias D, Olofsson Bagge R. Response and toxicity of repeated isolated limb perfusion (reILP) for patients with in-transit metastases of malignant melanoma. Ann Surg Oncol. 2019;26(4):1055-1062. doi:10.1245/s10434-018-07143-4
23. Duarte C, López H. Melanoma acral lentiginoso, revision bibliografica. Rev Colomb Cirugía. 2014;29(2):155-166.

24. Mckinnon JG. Palliation by Perfusion: Is it Worth it ? J Surg Oncol. 2005;91:217-218. doi:10.1002/jso.20316

25. Cornett WR, Mccall LM, Petersen RP, et al. Randomized Multicenter Trial of Hyperthermic Isolated Limb Perfusion With Melphalan Alone Compared With Melphalan Plus Tumor Necrosis Factor : American College of Surgeons Oncology Group Trial Z0020. J Clin Oncol. 2006;24(25):41964201. doi:10.1200/JCO.2005.05.5152

26. Klaase JM, Kroon BBR, Van Geel BN, Eggermont AMM, Franklin HR, Hart GAM. Patient and treatment related factors associated with acute regional toxicity after isolated perfusion for melanoma of the extremities. Am J Surg. 1994;167:618-620.

27. Deroose JP, Eggermont AMM, Geel AN Van, Wilt JHW De, Burger JWA, Verhoef C. 20 Years Experience of TNF-Based Isolated Limb Perfusion for In-Transit Melanoma Metastases: TNF Dose Matters. Ann Surg Oncol. 2012;19:627-635. doi:10.1245/s10434-011-2030-7

28. Grünhagen D, de Wilt JHW, Van Geel AN, Eggermont AMM. Isolated limb perfusion for melanoma patients - a review of its indications and the role of tumour necrosis factor- a. EJSO. 2006;32:371-380. doi:10.1016/j.ejso.2006.01.015

29. Fraker DL, Alexander HR, Andrich M, Rosenberg SA. Treatment of patients with melanoma of the extremity using hyperthermic isolated limb perfusion with melphalan, tumor necrosis factor, and interferon gamma: results of a tumor necrosis factor dose-escalation study. J Clin Oncol. 1996;14(2):479-489. doi:10.1200/JCO.1996.14.2.479

30. Lienard D, Ewalenko P, Delmotte J, Renard N, Lejeune FJ. High-Dose Recombinant Tumor Necrosis Factor alpha in Combination With Interferon gamma and Melphalan in Isolation Perfusion of the Limbs for Melanoma and Sarcoma. J Clin Oncol. 1992;10(1):52-60.

31. Grünhagen BDJ, Verhoef C. Isolated Limb Perfusion for Stage III Melanoma : Does It Still Have a Role in the Present Era of Effective Systemic Therapy ?: Page 2 of 2. Oncol J. 2016;30(12):1045-1052.

32. Johansson J, Kiffin R, Andersson A, et al. Isolated Limb Perfusion With Melphalan Triggers Immune Activation in Melanoma Patients. Front Oncol. 2018;8(December):1-11. doi:10.3389/fonc.2018.00570

33. Read RL, Thompson JF. Managing in-transit melanoma metastases in the new era of effective systemic therapies for melanoma. Expert Rev Clin Pharmacol. 2019;12(12):1107-1119. doi:10.1080/17512433.2019. 1689121

34. Teras J, Kroon HM, Thompson JF, et al. First Eastern European experience of isolated limb infusion for in-transit metastatic melanoma confined to the limb: is it still an effective treatment option in the modern era? Eur J Surg Oncol. 2020;46(2):272-276. doi:10.1016/j. ejso.2019.10.039

35. Ekenberg M, Wesslau H, Bagge RO, Engström M. Patient experiences with isolated limb perfusion for malignant melanoma - a qualitative study. Eur J Oncol Nurs. 2019;43:101672. doi:10.1016/j.ejon.2019.101672 